\title{
A Machine Learning-based Approach for Land Cover Change Detection Using Remote Sensing and Radiometric Measurements
}

\author{
Nabil Zerrouki, Fouzi Harrou, Member, IEEE, Ying Sun, Lotfi Hocini
}

\begin{abstract}
An approach combining the Hotelling $T^{2}$ control method with a weighted random forest classifier is proposed and used in the context of detecting land cover changes via remote sensing and radiometric measurements. Hotelling $T^{2}$ procedure is introduced to identify features corresponding to changed areas. Nevertheless, $T^{2}$ scheme is not able to separate real from false changes. To tackle this limitation, the weighted random forest algorithm, which is an efficient classification technique for imbalanced problems, has been successfully applied to the features of the detected pixels to recognize the type of change. The feasibility of the proposed procedure is verified using SZTAKI AirChange benchmark data. Results proclaim that the proposed detection scheme succeeds to effectively identify land cover changes. Also, the comparisons with other methods (i.e., neural network, random forest, support vector machine, and $k$-nearest neighbors) highlight the superiority of the proposed method.
\end{abstract}

Index Terms-Land cover change detection, multi-spectral sensors, multi-date measurements; remote sensing, multivariate statistical approach, Random Forest classification.

\section{INTRODUCTION}

Remote sensing is becoming essential for environmental protection, monitoring, and soil mapping throughout the last few decades [1], [2]. Compared to conventional mapping techniques, like photo-interpretation, spatial remote sensing (passive/ active) has incontestable advantages in terms of cost, speed, and the covered area [2]-[4]. In particular, satellite remote sensing plays a crucial role in agriculture management and planning, which is an important pillar for economic growth of countries [5], [6].

All over the years, strenuous efforts have been invested in research and industry and designing numerous satellitemounted sensors to evaluate the spatiotemporal evolution of crop conditions [7]. The developed satellite-based sensors, which have the desirable characteristics (spectral and temporal) for appropriately monitoring the crop acreage, include

N. Zerrouki is with University of Sciences and Technology Houari Boumédienne, LCPTS Faculty of Electronics and Computer Science, Algiers, and DIIM Laboratory, Center for Development of Advanced Technology (CDTA), Baba-Hassen, Algiers, Algeria.

F. Harrou and Y. Sun are with King Abdullah University of Science and Technology (KAUST) Computer, Electrical and Mathematical Sciences and Engineering (CEMSE) Division, Thuwal, 23955-6900, Saudi Arabia e-mail: fouzi.harrou@kaust.edu.sa

L. Hocini is with Mouloud Mammeri University (UMMTO), Departement of Electronics, Faculty of Electronic Engineering and Computer Science, Laboratory of Analysis and Modeling of the Random Phenomena, BP 17 RP, Tizi-Ouzou, and DIIM Laboratory, Center for Development of Advanced Technology (CDTA), Baba-Hassen, Algiers, Algeria.
LANDSAT-TM, Synthetic Aperture Radar (SAR), ENVISAT ASAR, RISAT-1, and MODIS [5]. Furthermore, with the development of advanced technologies in remote sensing, rich measurements sources are available now for crop monitoring and classification [6]. Various methods have been designed for crop monitoring and mapping based on SAR data [8]. For instance, Zhao et al. [9] investigated the feasibility of c-and $\mathrm{x}$-band polarimetric SAR (PolSAR) images to analyse and discriminate crop harvest patterns using a decision tree approach. In [10], time series of C- and X- band PolSAR data have used for detecting grassland cutting practices. Other researchers focused on using a time series of vegetation indices, such as normalized difference vegetation index (NDVI), which is an extensively used index in vegetation monitoring [5].

Land cover change detection (LCCD) is one of the most imperative issues in remote sensing, which can help to understand landscape dynamics [3]. Moreover, LCCD is useful in other applications, such as in burned areas assessment, study of shifting cultivation, pollution monitoring, and deforestation and desertification valuation [3]. In addition to soil mapping, LCCD allows an automatic measure of change variations in a specific region. In fact, LCCD can be used for various purposes including monitoring and management of pollution [11], desertification [12], and deforestation [13]. Recently, several LCCD techniques have been proposed in the literature [14][17]. In [14], Zhu et al. proposed a change detection and classification algorithm based on threshold derived from all spectral bands of the Landsat data. In such an approach, pixels are assigned to the changed class when the difference between observed and predicted images exceeds a threshold value. $\mathrm{Fu}$ et al. proposed a change detection system for urban growing monitoring using thermal infrared data onboard Landsat sensors [15]. In [16], Mayes et al. introduced a linear spectral mixture analysis approach with Landsat data applied for forest changes assessment. In [17], Espinoza-Molina et al. used local features extracted from spectral indices using a clustering algorithm. The LCCD is then performed by counting the frequency of the assignments of a changed pixel to a specific class along the time series. In [18], Jia-cun et al. proposed an object change detection method using high spatial resolution remote sensing imagery. In this approach, the image was first segmented into different objects and then a supervised classification based on a neural network was applied to detect changed objects. It is worth understanding that numerous change detection systems have been proposed in the literature. 
However, several proposed systems in the literature classify false changes as changed regions. Indeed, many phenomena such as weather and vegetation growth can lead to results with false changes. Furthermore, many proposed systems do not consider that change detection is an imbalanced problem, where changed class constitutes only a reduced number of samples compared to the unchanged class.

In a previous work [4], the authors introduced a strategy based on a multivariate exponentially weighted moving average scheme and SVM classification for LCCD. Based on the overall accuracy as an evaluation metric, this approach showed good capacity in separating real changes from false changes. However, in the design of this approach, the problem of imbalanced data is ignored. While the overall accuracy is high but it does not reflect the classification quality of the minority class (i.e., changed class). As pointed out in the literature, SVM classifier provides unsuitable classification performance when applied to imbalanced datasets [19], [20]. Accordingly, this paper focuses on detecting and identifying land cover changes by considering the problem of imbalanced datasets.

Thereby, it is necessary to consider the imbalanced classification to further enhance the detection of LCCs. Hence, in the present study, an effective methodology to monitor land cover changes using radiometric features is presented. The intensity of radiometric data is obtained from passive sensors (images acquired in visible wavelength). It should be noticed that LCCD based on remote sensing data remains a challenging problem due to different phenomena like shadows of clouds which can change the values of the radiometric data and consequently deliver poor or misleading results. Accordingly, it is crucial to design an effective approach to appropriately identify land-cover changes. Towards this end, an approach amalgamating the benefits of the Hotelling $T^{2}$ monitoring test and those of the weighted random forest algorithm (WRF) classifier is designed to suitably detect and identify land cover changes. The advantage of the $T^{2}$ scheme is its ability to monitor multivariate data simultaneously and its implementation is an easy task. However, real and false (reversible) changes are not separable by using the $T^{2}$ scheme. This means that these two types of changes may have relatively similar features when the $T^{2}$ is used. To bypass this limitation, WRF algorithm, which is an efficient classifier to deal with imbalanced data classification [21], is used to discover the dissimilarity between real changes and false changes. Specifically, only features of selected pixels during the detection phase via $T^{2}$ scheme are used by WRF. In other words, the role of the detection phase is to remove features without change, hence the size of the training dataset in WRF is reduced. In this approach, the WRF classifier focuses only on discriminating real changed from false changed pixels. In fact, in practice, false changed pixels are classification error prone. The feasibility of the proposed strategy is verified using data from the SZTAKI Air Change benchmark. The results indicate that the integrated $T^{2}$-WRF strategy is found to be suitable in detecting real changes with a reduced error rate. Also, the proposed approach is compared to several conventional algorithms (i.e., neural network, ran- dom forest, support vector machine, and $k$-nearest neighbors) and demonstrated enhanced performance. The next section presents radiometric feature extraction. $T^{2}$-based LCCD and WRF classification are described in Sections III and IV respectively. In Section V we assess the efficiency of the developed approach, and conclusions are presented in Section VI.

\section{Features EXTRACTION FOR LCCD}

Extracting important features from remote sensing data is not an obvious task due to the presence of several regional characteristics, such as build areas and large areas with vegetation. In addition, with the high level of similarities between some thematic classes of pixels, feature extraction becomes more challenging. For instance, 'less dense urban' and 'bare soil' have relatively comparable features, particularly in the case of limited spatial resolution of the remotely sensed imagery. In traditional change detection technologies, feature generation is accomplished via a software, like ENVI1 or eCongnition2.

In this paper, we associate to the same pixel at different time points (at $t 1$ and $t 2$ ) an observation sequence that represents a series of radiometric vectors in three bands. So, each pixel is represented by six features, $\left(f_{1}, \ldots, f_{6}\right)$ (Figure 1$)$. In fact, the level of variation in the pixels radiometric values can be used as an indicator to identify the type of change. For example, in the case of a disaster, the image exhibits a high radiometric change, whereas, in the case of growing urban, the radiometric variation is rather smooth.

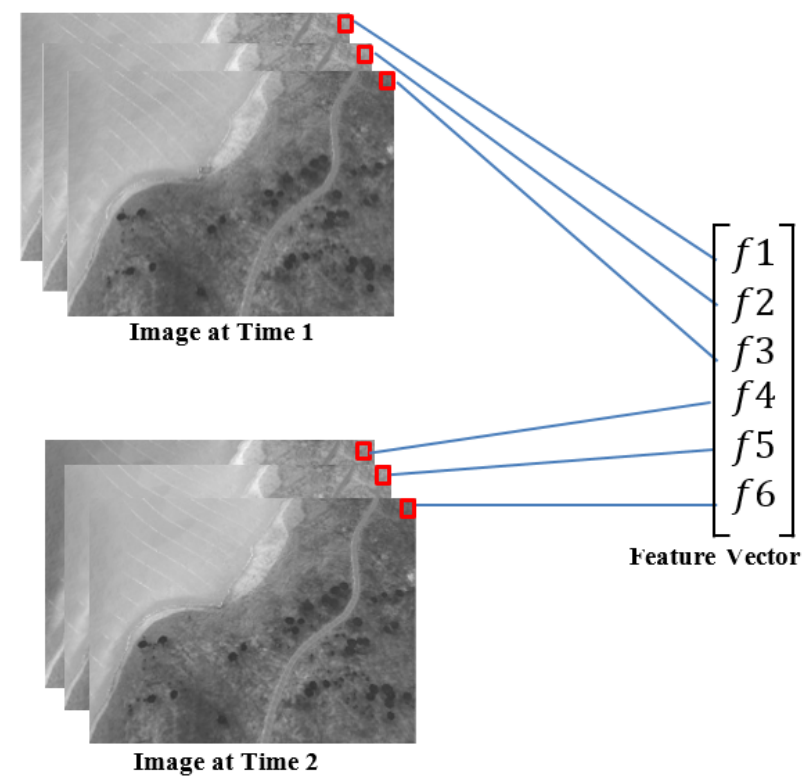

Fig. 1. Sample of remote sensing feature extraction at different times, $t 1$ and $t 2$, when considering three bands.

With the presence of potential changes in remote sensing measurements, the goal is to appropriately classify real and false changes. For this purpose, we develop two stages procedure including the detection and classification of changes (Figure 2). The detection stage consists of the application of the $T^{2}$ scheme on radiometric data to identify changed areas. 
In other words, the detection phase allows a separation of unchanged pixels from changed pixels. On the other hand, the WRF-based classification procedure focuses only on the features of the pixels previously detected by $T^{2}$ (considered as changed). The main advantage of using two-stages-based approach (detection followed by classification) is to reduce the number of training samples used by the WRF algorithm compared to classification without a detection phase. Unchanged pixels, which are identified by the $T^{2}$ scheme, have been excluded from the classification stage.

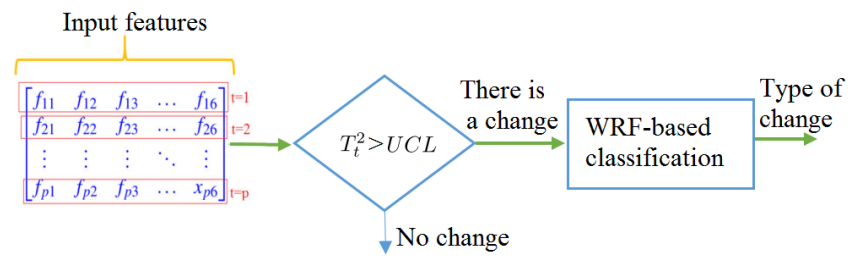

Fig. 2. A block diagram of the proposed detection mechanism.

\section{Hotelling $T^{2}$ MONITORING SCHEME}

The $T^{2}$ approach is the most common multivariate process monitoring technique used to monitor the mean of a normally distributed process [22], [23]. Consider a data matrix $\mathbf{X} \in R^{n \times m}$ with $n$ measurements and $m$ process variables, $\mathbf{X}=\left[\mathbf{x}_{1}^{T}, \ldots, \mathbf{x}_{n}^{T}\right]^{T}$. Assume $\mathbf{x}_{t}, t=1,2, \ldots, n$ is normally distributed with mean $\mu_{0}$ and covariance matrix $\boldsymbol{\Sigma}_{0}$. A multivariate Shewhart chart, also known as a $T^{2}$ chart or a $\chi^{2}$ chart [24], to monitor the process mean is based on the decision statistic:

$$
T_{t}^{2}=\left[\left(\mathbf{x}_{t}-\mu_{0}\right)^{T} \boldsymbol{\Sigma}_{0}^{-1}\left(\mathbf{x}_{t}-\mu_{0}\right)\right],
$$

where $\mathbf{x}_{t}$ is a vector of $m$ variables, $\mu_{0}$ is a vector of in-control means of each variable, and $\boldsymbol{\Sigma}_{0}$ is the covariance matrix of $\mathrm{X}$.

In cases when both $\mu_{0}$ and $\boldsymbol{\Sigma}_{0}$ are unknown, they can be estimated by the empirical mean vector, $\overline{\mathbf{X}}$, and the empirical covariance matrix $\mathbf{S}$. Then, the maximum likelihood estimators of $\mu_{0}$ and $\boldsymbol{\Sigma}_{0}$ are:

$$
\begin{gathered}
\widehat{\mu}=\overline{\mathbf{X}}=\frac{1}{n} \sum_{i=1}^{n} X_{i}, \\
\widehat{\mathbf{\Sigma}}=\frac{1}{n} \sum_{i=1}^{n}\left(X_{i}-\overline{\mathbf{X}}\right)\left(X_{i}-\overline{\mathbf{X}}\right)^{T} .
\end{gathered}
$$

We claim that there is a change when

$$
T_{t}^{2}>U C L
$$

where $U C L=\chi_{\alpha, m}^{2}$ is the upper limit for the $T^{2}$ control chart, and $\alpha$ denote a chosen significance level [25]. In cases when the data distribution is non-normal or unknown, the distribution of $T^{2}$ statistic can be estimated by using the univariate kernel density estimation (KDE) [26]. Then, a nonparametric threshold of $T^{2}$ approach is defined as the $(1-\alpha)$-th quantile of the estimated distribution of $T^{2}$ statistic obtained by KDE.

\section{LAND-COVER CLASSIFICATION PHASE VIA WRF}

Land-cover classification aims at separating real from false (reversible) changes for the detected pixels. The use of the detection phase permits to reduce the number of pixels to be classified, where unchanged pixels are excluded from the classification phase. The key concept in classification is to define the model's parameters in the training phase. After the model is determined, then it will be employed to classify new data. Here, we choose the weighted random forest procedure as classifier because of its capability to operate efficiently with large and imbalanced datasets. Furthermore, it can handle an important number of features without overfitting (by its capacity to manage an important number of trees).

It should be pointed out that Random forest classier can reach a high accuracy than many conventional classification algorithms, where it minimizes the overall classification error rate and has the capacity to merge many classifiers [27]-[29]. However, in the presence of imbalanced data RF ability is slightly weakened. This is because that each classifier has the same weight before combination (voting phase) [30]. Hence, to address this problem, a methodology of merging classification algorithms with different weights has been developed. In the WRF approach, each individual classifier is pondered by a weight (representing the individual confidence of its decisions) [31]. Class weights are used to permit a robust separation between the majority and the minority classes. In other words, a larger weight is given to the minority class, hence a higher misclassification cost for changed pixels.

The key concept of the WRF classifier is to compute the misclassification rate for each tree Out-Of-Bag (OOB), and the class prediction for WRF is then given by combining the weighted vote from each individual tree. Instead, algorithms based on error minimization like Support Vector Machine or neural network, WRF has an effective methodology for estimating classes when a proportion of the data is missing (imbalanced problems) [31].

Figure 3 illustrates a diagrammatic representation of the weighted random forest. Initially, the algorithm assigns a weight to each class, where the minority class attributed higher weight. The class weights, $C W(j)$, are introduced in two places of the algorithm namely (i) in the tree induction procedure to find tree's splits, (ii) and in the terminal nodes, $F n(i)$, of each tree whose impact the guidance of the voting process.

Initially, a set of new training subsets $S(i)$ are generated randomly from the original training set using bootstrap sampling technique. The same strategy is applied to each subset (boostraping), where only $\sqrt{M}$ features are picked from the extracted features ( $M$ represents the total number of features). The new generated features are then predicted separately using different trees for each subsample. The remaining samples after each boostrap sampling are used for tree's evaluation procedure. Only tree corresponding to the best accuracy is selected. In fact, the class weights are considered in the tree selection during separate prediction phase. The next step consists of aggregating the results of all the trees. This procedure is based 


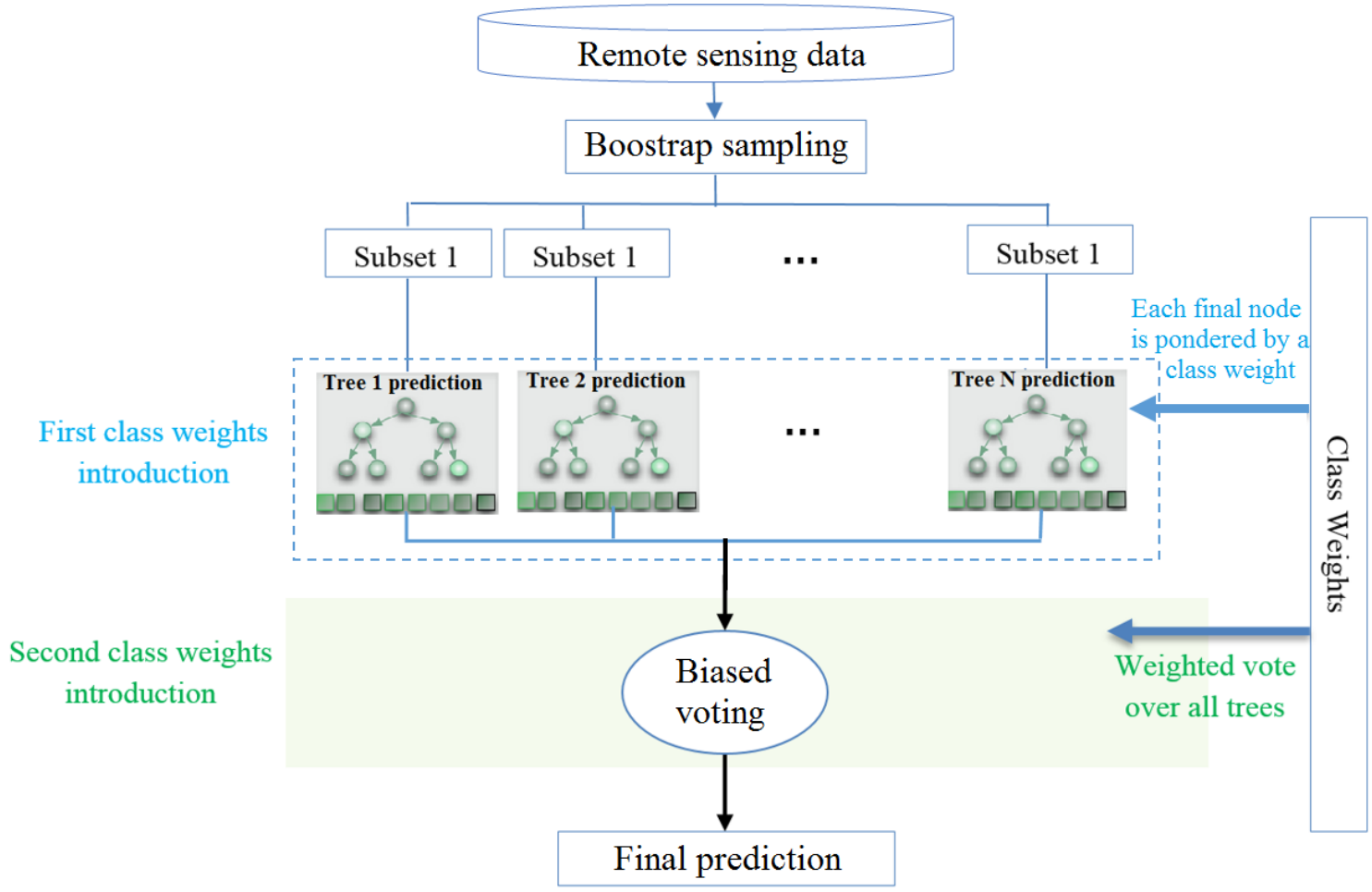

Fig. 3. A block diagram of the WRF classifier.

on weighted voting procedure $V(j)$ instead of the majority voting to allow an adequate separation between the majority and the minority classes. The weighted votes are expressed as follows:

$$
V(j)=\sum_{i=1}^{N} \frac{F n(i)}{\sum_{j=1}^{k} C W(k)},
$$

where $N$ and $K$ represent the number of trees and the number classes, respectively

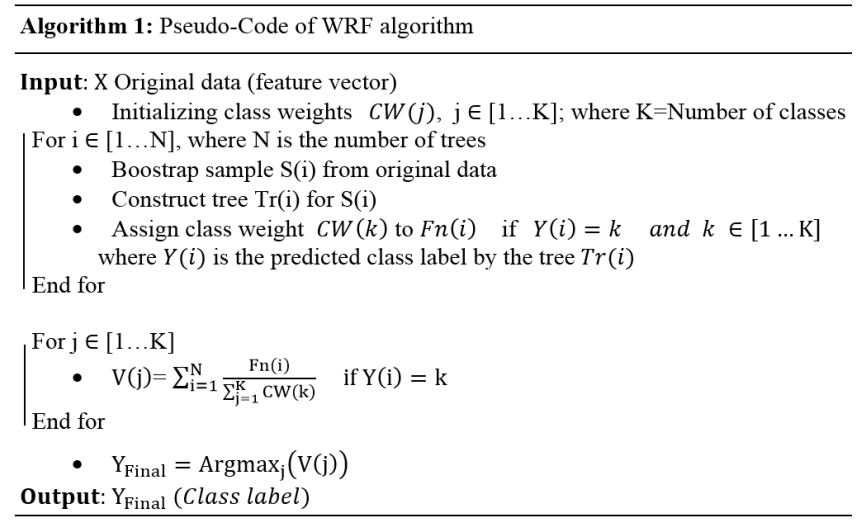

\section{RESULTS AND DISCUSSION}

\section{A. Data collection}

This part is dedicated to verifying the effectiveness of the $T^{2}$-WRF approach in detecting LCCs by using real multi-dateimages. Data from SZTAKI AirChange Benchmark set [32] has been used to investigate the capacity of the proposed approach. This dataset is gathered by the Hungarian Institute of Geodesy Cartography and Remote Sensing (HIGCRS), and consisting of several optic aerial image pairs with a resolution of 1.5 meters of $952 \times 640$ pixels [32]. The SZTAKI database is structured into three subsets: SZADA, TISZADOB, and ARCHIVE. The multi-date images are associated with the ground of truth masks. They are acquired at different time intervals to include different changes such as deforestation assessment and urban growing planning. Figure 4(a-c) depicts an example of the image pairs at two time instant $(t 1$ and $t 2$ ) the corresponding binary mask, highlighting changed areas with white dots. (a)

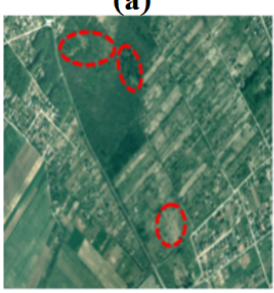

(b)

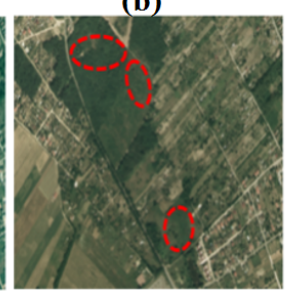

(c)

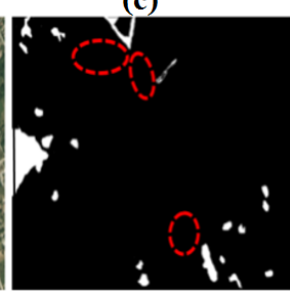

Fig. 4. Sample of an image at $t 1$ (a), same image at $t 2$ (b), and the ground truth image (c).

The SZTAKI AirChange Benchmark, which is a rich data, covers the whole thematic classes and contains different regional physiognomies like vegetation (i.e., agricultural areas known by homogenous regions) and urban areas (generally cities and towns known by heterogeneous areas). This variety of data makes the change detection a challenging task, in particular in the ground truth where reversible changes are 
labeled as unchanged pixels. To address this difficulty, three main categories of pixels are considered here: unchanged, real changes and false changes. The first class presents irreversible changes like desertification and natural phenomena (e.g., forest fires). The unchanged class contains pixels that keep the same thematic classes across the time interval, such as water areas. The third class, false change is for pixels that have undergone a change in radiometric values but not in the thematic classes such as variation in the degree of greenery for grass areas or snowfall. This type of change is not labeled as changed because it is seasonal.

\section{B. Evaluating LCCD Results}

To evaluate the performance of $T^{2}$ monitoring scheme, we first used change-free data to compute the $T^{2}$ decision threshold. The training data consists of the variation between radiometric values in different bands collected at two different instants. The data consist of three columns corresponding to the number of bands and 500 rows (representing the number of pixels). Based on the training data, the threshold is set to be $U C L=21.03$ with the predefined probability of false alarm of $5 \%$. Here, the training data has been chosen from different areas including bare soil, vegetation, and urban regions, to consider the variety of land cover. To illustrate the detection capability of the $T^{2}$ approach, we used testing data of 500 pixels comprising real changes. Results of the $T^{2}$ approach is presented in Figure 5(a), which indicates the presence of possible LCC. The $T^{2}$ approach based on testing data with false changes is displayed in Figure 5(b). We conclude that this multivariate approach is able to detect changes but it cannot differentiate between true and false changes.

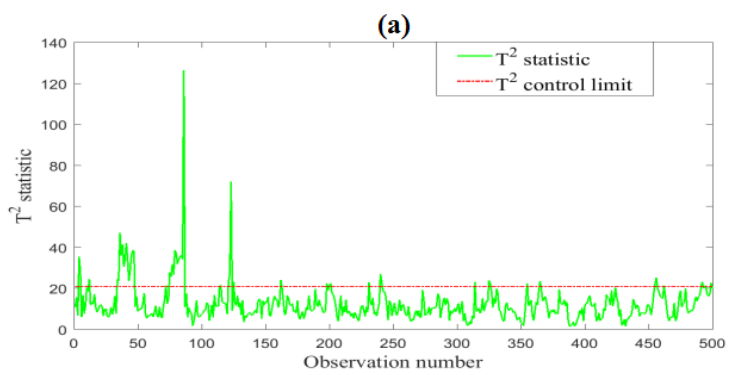

(b)

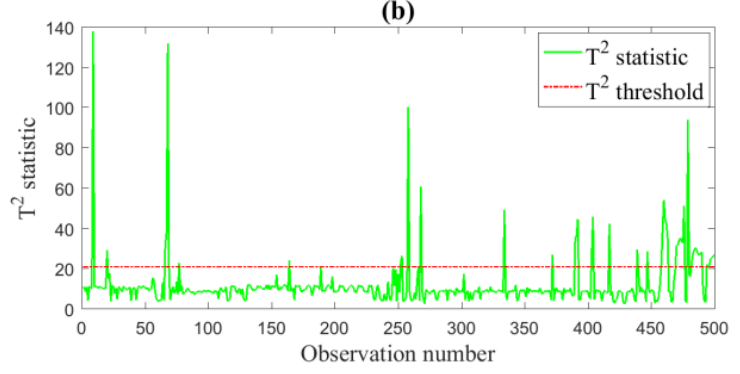

Fig. 5. $T^{2}$ approach: (a) real and (b) false changes.

To cope with this problem, the WRF classifier is used to separate real from false changes. The WRF is applied only to the features of the flagged pixels by $T^{2}$ approach. The proposed $T^{2}-\mathrm{WRF}$ strategy is compared with some common machine learning schemes namely neural network, Random Forest, $k$-NN, and SVM without a detection step.

The parameters of each algorithm, which provide the highest accuracy, have been determined in the training stage. The NN classifier with one hidden layer and nine neurons has been selected. The number of neighbors in $k$-NN is set to 3 . In SVM, radial basis function (RBF) kernel with the width of the Gaussian kernel $\sigma=0.125$, and the parameter for the soft margin cost function $C=128$, is used. For the WRF classifier, after testing different parameter settings, we fix the number of trees in the forest to 10 and weights to 15 . Since change detection presents a case of imbalanced data, so, to accord more interest to the rare class (changed) two accuracies corresponding to changed and unchanged classes are separately computed for different algorithms.

\section{Evaluation metrics}

To evaluate quantitatively the efficiency of the proposed procedure $T^{2}-\mathrm{WRF}$, different evaluation measures have been computed from the obtained results namely: overall accuracy, Kappa coefficient, and F-measure.

- The overall accuracy reflects a comparison between reference pixels and classified pixels. It represents the percentage of correctly classified pixels. This ratio is calculated from the confusion matrix using the mean of the diagonal elements. It is expressed as:

$$
\text { Accuracy }=\frac{(T P+T N)}{(F P+T P+T N+F N)},
$$

where $\mathrm{TP}$ is true positives, $\mathrm{TN}$ is true negatives, FP is false positives, and $\mathrm{FN}$ is false negatives.

- The second evaluation measure used in this paper is Kappa coefficient. This statistical measure is more relevant than overall accuracy since it takes into account pixels correctly classified by chance. This metric is expressed as:

$$
\text { Kappa }=(P(a)-P(e))(1-P(e))
$$

where $P(a)$ and $P(e)$ are the probability of relative agreement and the probability of chance agreement, respectively.

- F-measure is another performance metric based on precision and recall. This metric represents the harmonic significance of precision (p) and recall ( $r$ ). It is expressed as follows:

$$
F=\left(1+\beta^{2}\right) \frac{(p \times r)}{\left(\left(\beta^{2} \times p\right)+r\right)},
$$

where $p=\frac{t p}{(t p+f p)}$ and $r=t p(t p+f n)$. The parameter $\beta$ is related to the different types of error $(\beta=1)$.

Figure 6 illustrates a sample of image pairs from the different subsets, namely ARCHIVE, TISZADOB, and SZADA at two points in time $t_{1}$ (column 1) and $t_{2}$ (column 2) with their corresponding ground truth and $T^{2}-\mathrm{WRF}$-based classification, which are represented in the third and the fourth column, respectively. The changed area is highlighted with white dots. 
The first subset (ARCHIVE) represents a comparison between two images taken in 1984 and 2007 respectively. This pair is very challenging since it contains different types of changes due to the long time (23 years) between the two scene shots. The second subset (TISZADOB) contains five multidate image pairs with their corresponding ground of truth. These image pairs are acquired between 2000 and 2007, respectively. This set of scenes covers a region of $6.8 \mathrm{KM} 2$ with a resolution of $1.5 \mathrm{~m}$. The last subset (SZADA) comprises seven image pairs with a change interval of five years (from 2000 to 2005) covering an area of 9.5 KM2. Each image pair has a dimension of $952 \times 640$ pixels and with a resolution of $1.5 \mathrm{~m}$.

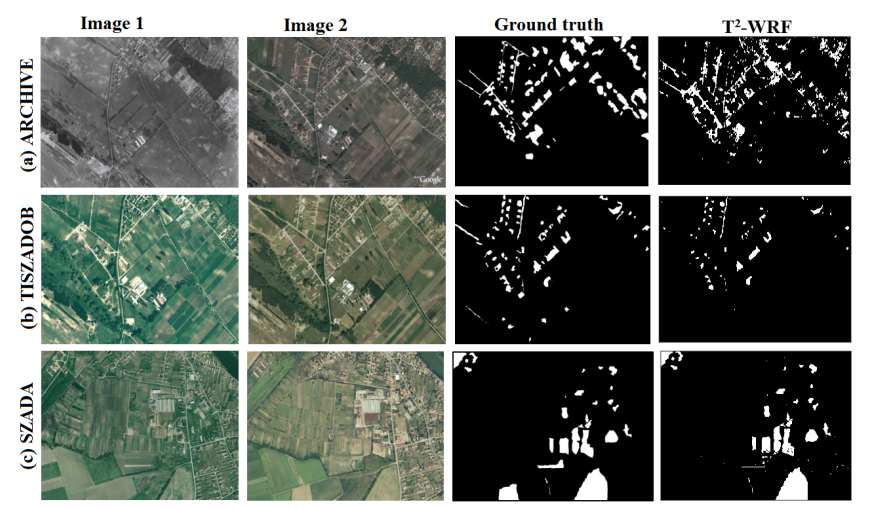

Fig. 6. Sample of an image at $t_{1}$ (column 1), same image at $t_{2}$ (column 2 ), the ground truth image (column 3 ), and change detection results of $T^{2}$ WRF approach (column 4) when applied to ARCHIVE (a), TISZADO (b), and SZADA (c), respectively.

Table I displays the detection performances of the proposed strategy when applied to different subsets of SZTAKI Air change database namely ARCHIVE, TISZADOB, and SZADA. Overall, results indicate the suitable capability of the proposed approach in identifying land cover changes. Also, it can be seen that the the lowest recognition accuracy is achieved when using ARCHIVE subset (Table I). This is mainly due to the degraded quality of the first image in the ARCHIVE subset, which was acquired in 1984, compared to the rest of the images.

TABLE I

DETECTION PERFORMANCE OF $T^{2}$-WRF APPROACH WHEN APPLIED ARCHIVE, TISZADOB, AND SZADA SUBSETS SEPARATELY.

\begin{tabular}{l|l|l|l|l|l|l}
\hline \multicolumn{2}{c|}{} & Accuracy & Sensitivity & Specificity & Precision & F-Measure \\
\hline \multirow{2}{*}{ Archive } & Changed & 0.8452 & 1.0000 & 0.8100 & 0.7750 & 0.8700 \\
\cline { 2 - 7 } & Unchanged & 0.9428 & 0.9162 & 1.0000 & 1.0000 & 0.9263 \\
\hline \multirow{2}{*}{ Tiszado o } & Changed & 0.8528 & 0.8632 & 0.0050 & 0.9497 & 0.9044 \\
\cline { 2 - 7 } & Unchanged & 0.9613 & 0.9649 & 0.0029 & 1.000 & 0.9522 \\
\hline \multirow{2}{*}{ Szada } & Changed & 0.8611 & 0.8823 & 0.0026 & 0.9557 & 0.9176 \\
\cline { 2 - 7 } & Unchanged & 0.9519 & 0.9531 & 1.0000 & 1.0000 & 0.9294 \\
\hline
\end{tabular}

Table II presents the classification results of the $T^{2}-\mathrm{WRF}$ strategy and the conventional procedures when applied to Sztaki Airchange dataset. As previously mentioned, the overall accuracy does not correctly reflect the robustness of the algorithm when it is about imbalanced problems. For this reason, we calculated the accuracy on each class (changed and unchanged) separately. The results given by $K-\mathrm{NN}$,
SVM, RF, neural network and MEWMA-SVM [4] algorithms are shown for comparison. Figure 7 displays the F-measure comparison between the studied approaches. The results in Table II and Figure 7 indicate that the $T^{2}$-WRF-based LCC detection strategy is demonstrated to be better in identifying LCCs in comparison to the conventional learning algorithms presented in this study. It can be noticed that for the unchanged class, there is not much difference between obtained accuracies SVM: $91.05 \% T^{2}-\mathrm{WRF}: 95.20 \%$. However, concerning changed class, the $T^{2}$-WRF has clearly outperformed the rest of change detection methods where $T^{2}-\mathrm{WRF}$ has an accuracy of $85.3 \%$ while SVM has $69.59 \%$ and RF $51.20 \%$. It can be concluded that the LCC detection is improved by integrating the desirable proprieties of $T^{2}$-based detection approach with WRF classification. Also, the conventional procedures (i.e., $\mathrm{NN}, k-\mathrm{NN}$, and SVM) resulted in many misclassifications (Table II). This is because pixels with false changes are misclassified as "changed". Furthermore, some classification algorithms such as Support Vector Machine or neural network are based on improving global accuracy by reducing the overall error. Accordingly, these methods ignore the class proportion or balance of classes (well-classifying majority class and completely misclassifying the minority class) which are not suitable in such situations (imbalanced data). Table II indicates that MEWMA-SVM produces a high accuracy for unchanged class, but it has low accuracy for changed class (i.e., 67.38\%). Also, MEWMA-SVM approach showed an acceptable capacity in separating changes with high overall accuracy since the percentage of pixels belonging to minority class is underrated compared to the majority class samples. This confirms the limitation of the SVM algorithm when applied to imbalanced datasets. Another reason that the $T^{2}$ WRF approach surpassed the other algorithms is that weighted random forest provides high accuracy over the minority class while keeping acceptable accuracy for the majority class.

TABLE II

COMPARISON OF THE $T^{2}$-WRF PROCEDURE WITH CONVENTIONAL MACHINE LEARNING APPROACHES.

\begin{tabular}{c|c|c|c}
\hline & $\begin{array}{c}\text { Accuracy } \\
\text { (changed class) }\end{array}$ & $\begin{array}{c}\text { Accuracy } \\
\text { (unchanged class) }\end{array}$ & Kappa \\
\hline Neural network & 66.53 & 90.92 & 0.76 \\
\hline$k$-NN & 41.21 & 86.66 & 0.72 \\
\hline SVM & 69.59 & 91.05 & 0.76 \\
\hline RF & 51.20 & 93.18 & 0.78 \\
\hline MEWMA-SVM & 67.38 & 95.58 & 0.80 \\
\hline WRF & 85.30 & 95.20 & 0.87 \\
\hline
\end{tabular}

Now, to highlight the benefit of amalgamating $T^{2}$ and WRF in detecting LCCs, comparisons with some proposed systems conducted on the SZTAKI AirChange data are reported in Tables III. The comparison is done with two powerful classifiers namely: Three Layer Markov Random Field [33] and Multilayer mixed Markov model [32]. To allow a fair comparison, only the overall accuracy is presented as comparison criterion instead of separate precisions of each class $(85.3 \%$ and $95.2 \%$ ) since the remaining methods [32], [33] do not consider the problem of imbalanced data (only overall accuracy is computed). One can notice that the overall accuracy 


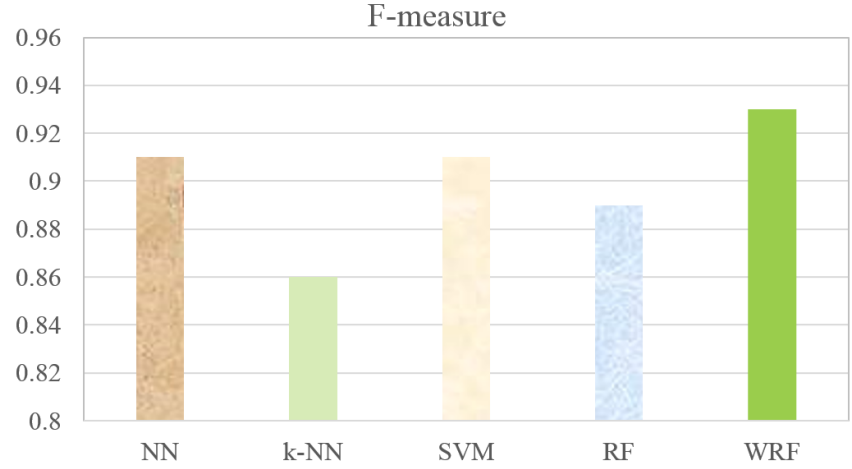

Fig. 7. F-measure comparison of the proposed approach and standalone NN, K-NN, SVM and RF.

of the proposed approach is too close to unchanged class accuracy (95.2\%) where it represents the majority samples compared to changed pixels (minority class) presenting an accuracy of $85.3 \%$. From Table III, It can be observed that the proposed $T^{2}-\mathrm{WRF}$ approach performs better in detecting changed regions than the two methods presented in [32], [33] (both approaches consider the sequential aspect of the data by the use of Markov formalism). This improvement is mainly due to the incorporation of the detection phase to separate unchanged pixels and the use of WRF algorithm to provide a final decision on detected pixels and separate real changes from false changed regions. Unlike the methods proposed in [32], [33], the decision is based on the classification step alone.

TABLE III

LCCS DETECTION PERFORMANCE OF DIFFERENT METHODS USING SZTAKI AIRCHANGE DATA.

\begin{tabular}{l|l|l}
\hline & Approach & Accuracy (\%) \\
\hline Singh et al. [33] & Three Layer Markov Random Field & 94.65 \\
\hline Benedek et al. [32] & Multilayer mixed Markov model & 95 \\
\hline Proposed approach & $T^{2}$-WRF & 95.20 \\
\hline
\end{tabular}

\section{CONCLUSION}

In this paper, we introduced an effective land-cover change detection (LCCD) technique that utilizes variation in radiometric values of aerial images. In this approach, $T^{2}$ monitoring scheme has been utilized for LCCD and the WRF classifier has been applied to distinguish the type of change. Using the SZTAKI Air Change benchmark dataset, we show the efficiency of $T^{2}-\mathrm{WRF}$ in detection land-cover changes. The results indicate that the integrated $T^{2}-\mathrm{WRF}$ is found to be better in LCCD in comparison to other commonly used algorithms.

As future work, we plan to apply the coupled $T^{2}$-WRF approach to hyperspectral images to further enhance the effectiveness of land cover change detection. Since hyperspectral imagery captures more narrow bands than multispectral in the same range of the electromagnetic spectrum which offers a better characterization of different thematic classes. Furthermore, it would be worthwhile to do more work on change detection with very noisy measurements.
Indeed, it might is hard extracting relevant information on land changes from highly noisy data. While satellite-based time series data provide detailed information on land cover changes, they can also be noisy due to clouds and aerosols, which mask important features in the data. Accordingly, noisy measurements may degrade the quality of change detection classification. To enhance the performance of the proposed approach under noisy conditions, it is intended to apply waveletbased multiscale filter, a powerful feature/noise separation tool for denoising.

\section{ACKNOWLEDGMENT}

This publication is based upon work supported by the King Abdullah University of Science and Technology (KAUST) Office of Sponsored Research (OSR) under Award No: OSR2015-CRG4-2582. The authors (Nabil Zerrouki and Lotfi Hocini) would like to thank the DIIM laboratory, Centre de Developpement des Technologies Avancees (CDTA) for the continued support during the research.

\section{REFERENCES}

[1] X. Li, Q. Liu, R. Yang, J. Wen, J. Zhang, E. Cai, and H. Zhang, "The combination of ground-sensing network and satellite remote sensing in huailai county," IEEE Sensors Journal, vol. 16, no. 10, pp. 3819-3826, 2016.

[2] S. Nag, T. Hewagama, G. T. Georgiev, B. Pasquale, S. Aslam, and C. K. Gatebe, "Multispectral snapshot imagers onboard small satellite formations for multi-angular remote sensing," IEEE Sensors Journal, vol. 17, no. 16, pp. 5252-5268, 2017.

[3] S. Jin, L. Yang, P. Danielson, C. Homer, J. Fry, and G. Xian, "A comprehensive change detection method for updating the national land cover database to circa 2011," Remote Sensing of Environment, vol. 132, pp. 159-175, 2013.

[4] N. Zerrouki, F. Harrou, and Y. Sun, "Statistical monitoring of changes to land cover,' IEEE Geoscience and Remote Sensing Letters, vol. 18, no. 12 , pp. 1-5, 2018.

[5] V. Tomar, V. P. Mandal, P. Srivastava, S. Patairiya, K. Singh, N. Ravisankar, N. Subash, and P. Kumar, "Rice equivalent crop yield assessment using modis sensors based mod13a1-ndvi data," IEEE Sensors Journal, vol. 14, no. 10, pp. 3599-3605, 2014.

[6] T. Zhou, J. Pan, P. Zhang, S. Wei, and T. Han, "Mapping winter wheat with multi-temporal sar and optical images in an urban agricultural region," Sensors, vol. 17, no. 6, p. 1210, 2017.

[7] W. S. McInnes, B. Smith, and G. J. McDermid, "Discriminating native and nonnative grasses in the dry mixedgrass prairie with modis ndvi time series," IEEE Journal of Selected Topics in Applied Earth Observations and Remote Sensing, vol. 8, no. 4, pp. 1395-1403, 2015.

[8] D. Lu and Q. Weng, "A survey of image classification methods and techniques for improving classification performance," International journal of Remote sensing, vol. 28, no. 5, pp. 823-870, 2007.

[9] L. Zhao, J. Yang, P. Li, and L. Zhang, "Characteristics analysis and classification of crop harvest patterns by exploiting high-frequency multipolarization sar data," IEEE Journal of Selected Topics in Applied Earth Observations and Remote Sensing, vol. 7, no. 9, pp. 3773-3783, 2014.

[10] K. Voormansik, T. Jagdhuber, K. Zalite, M. Noorma, and I. Hajnsek, "Observations of cutting practices in agricultural grasslands using polarimetric sar," IEEE Journal of Selected Topics in Applied Earth Observations and Remote Sensing, vol. 9, no. 4, pp. 1382-1396, 2016.

[11] P. Zhang, Z. Lv, and W. Shi, "Local spectrum-trend similarity approach for detecting land-cover change by using SPOT-5 satellite images," IEEE Geoscience and Remote Sensing Letters, vol. 11, no. 4, pp. 738-742, 2014.

[12] F. Ling, W. Li, Y. Du, and X. Li, "Land cover change mapping at the subpixel scale with different spatial-resolution remotely sensed imagery," IEEE Geoscience and Remote Sensing Letters, vol. 8, no. 1, pp. 182186, 2011. 
[13] J. Inglada and G. Mercier, "A new statistical similarity measure for change detection in multitemporal sar images and its extension to multiscale change analysis," IEEE transactions on geoscience and remote sensing, vol. 45, no. 5, pp. 1432-1445, 2007.

[14] Z. Zhu and C. E. Woodcock, "Continuous change detection and classification of land cover using all available landsat data," Remote sensing of Environment, vol. 144, pp. 152-171, 2014.

[15] P. Fu and Q. Weng, "A time series analysis of urbanization induced land use and land cover change and its impact on land surface temperature with landsat imagery," Remote Sensing of Environment, vol. 175, pp. 205-214, 2016.

[16] M. T. Mayes, J. F. Mustard, and J. M. Melillo, "Forest cover change in miombo woodlands: modeling land cover of african dry tropical forests with linear spectral mixture analysis," Remote sensing of environment, vol. 165, pp. 203-215, 2015.

[17] D. Espinoza-Molina, R. Bahmanyar, R. Díaz-Delgado, J. Bustamante, and M. Datcu, "Land-cover change detection using local feature descriptors extracted from spectral indices," in Geoscience and Remote Sensing Symposium (IGARSS), 2017 IEEE International. IEEE, 2017, pp. 1938-1941.

[18] L. Jia-Cun, Q. Shao-Meng, and C. Xue, "Object-oriented method of land cover change detection approach using high spatial resolution remote sensing data," in Geoscience and Remote Sensing Symposium, 2003. IGARSS'03. Proceedings. 2003 IEEE International, vol. 5. Ieee, 2003, pp. 3005-3007.

[19] R. Akbani, S. Kwek, and N. Japkowicz, "Applying support vector machines to imbalanced datasets," in European conference on machine learning. Springer, 2004, pp. 39-50.

[20] R. Batuwita and V. Palade, "Class imbalance learning methods for support vector machines," Imbalanced learning: Foundations, algorithms, and applications, vol. 83, 2013.

[21] C. Chen, A. Liaw, and L. Breiman, "Using random forest to learn imbalanced data," University of California, Berkeley, vol. 110, pp. 1-12, 2004.

[22] F. Harrou, Y. Sun, M. Madakyaru, and B. Bouyedou, "An improved multivariate chart using partial least squares with continuous ranked probability score," IEEE Sensors Journal, vol. 18, no. 16, pp. 67156726, 2018.

[23] T. Cheng, F. Harrou, Y. Sun, and T. O. Leiknes, "Monitoring influent measurements at water resource recovery facility using data-driven soft sensor approach," IEEE Sensors Journal, vol. 19, no. 1, pp. 1-12, 2018.

[24] H. Hotelling, "Multivariate quality control illustrated by the air testing of sample bomb sights, Techniques of Statistical Analysis, Ch. II."

[25] _ "Analysis of a complex of statistical variables into principal components," Journal of Educational Psychology, vol. 24, pp. 417-441, 1933.

[26] E. Martin and A. Morris, "Non-parametric confidence bounds for process performance monitoring charts," Journal of Process Control, vol. 6, no. 6, pp. 349-358, 1996.

[27] Q. Wu, Y. Ye, Y. Liu, and M. K. Ng, "SNP selection and classification of genome-wide SNP data using stratified sampling random forests," IEEE transactions on nanobioscience, vol. 11, no. 3, pp. 216-227, 2012.

[28] Q. Li, Y. Gu, and N.-F. Wang, "Application of random forest classifier by means of a QCM-based e-nose in the identification of Chinese liquor flavors," IEEE Sensors Journal, vol. 17, no. 6, pp. 1788-1794, 2017.

[29] Z. Wang, Q. Zhang, J. Xiong, M. Xiao, G. Sun, and J. He, "Fault diagnosis of a rolling bearing using wavelet packet denoising and random forests," IEEE Sensors Journal, vol. 17, no. 17, pp. 5581-5588, 2017.

[30] B. Xia, H. Jiang, H. Liu, and D. Yi, "A novel hepatocellular carcinoma image classification method based on voting ranking random forests," Computational and mathematical methods in medicine, vol. 2016, 2016.

[31] J. Maudes, J. J. Rodríguez, C. García-Osorio, and N. García-Pedrajas, "Random feature weights for decision tree ensemble construction," Information Fusion, vol. 13, no. 1, pp. 20-30, 2012.

[32] C. Benedek and T. Szirányi, "Change detection in optical aerial images by a multilayer conditional mixed markov model," IEEE Transactions on Geoscience and Remote Sensing, vol. 47, no. 10, pp. 3416-3430, 2009.

[33] P. Singh, Z. Kato, and J. Zerubia, "A multilayer markovian model for change detection in aerial image pairs with large time differences," in Pattern Recognition (ICPR), 2014 22nd International Conference on. IEEE, 2014, pp. 924-929. 